\title{
Real-World Clinical Effectiveness of Liraglutide in Individuals 65 Years and Older with Type 2 Diabetes in the United States
}

\section{Abhishek Chitnis ${ }^{1 *}$, Michael L Ganz ${ }^{1}$, Mette Hammer ${ }^{2}$ and Jakob Langer ${ }^{2}$}

${ }^{1}$ Evidera, Lexington, MA, USA

${ }^{2}$ Novo Nordisk Inc., Plainsboro, NJ, USA

\begin{abstract}
Objective: Clinical trials have shown that liraglutide effectively lowers A1C levels and helps manage weight in Type 2 diabetes (T2D). Currently, no studies have solely evaluated the real-world clinical effectiveness of liraglutide in a T2D population of individuals 65 years and older. We examined the clinical effectiveness of liraglutide in individuals aged 65 years and older with T2D 6 and 12 months after starting therapy.

Methods: In this retrospective cohort study we used the General Electric Centricity electronic medical records database. We included individuals with T2D aged 65 years and older who initiated liraglutide at any time from January 1, 2010 to January 31, 2013. Individuals using either insulin or a glucagon-like peptide-1 analogue during 12 months before initiating liraglutide were excluded. Changes in $\mathrm{A} 1 \mathrm{C}$, weight, the proportion of individuals achieving a target $\mathrm{A} 1 \mathrm{C}<7 \%$ and occurrence of severe hypoglycemic events at 6 and 12 months were evaluated.

Results: We identified 517 individuals with T2D who were 65 years and older with $\mathrm{A} 1 \mathrm{C}>7 \%$ at baseline ( 45 days prior to therapy initiation to 7 days after). Individuals were, on average (standard deviation, SD), 70.6 (4.7) years old, $52.6 \%$ were female and $71.6 \%$ were white. A1C and weight at baseline were $8.2 \%(1.0 \%)$ and $101.0(19.7) \mathrm{kg}$ [222.6 (43.3) lbs], respectively. After 6 months A1C decreased by $0.76 \%(1.1 \%)$, weight decreased by $2.9(5.4) \mathrm{kg}$ [6.4 (11.9) lbs], and $37.1 \%$ of the sample achieved the target $\mathrm{A} 1 \mathrm{C}<7 \%$. Twelve-month outcomes were similar: $0.78 \%$ $(1.2 \%)$ reduction in A1C, 3.1 (5.7) $\mathrm{kg}[6.8(12.5) \mathrm{lbs}]$ reduction in weight, and $39.9 \%$ achieved $\mathrm{A} 1 \mathrm{C}<7 \%$. No evidence of severe hypoglycemia was found either at 6 or at 12 months.
\end{abstract}

Conclusion: Individuals with T2D 65 years and older experienced a significant and sustained reduction in A1C and weight over 6 and 12 months without any occurrence of severe hypoglycemia.

Keywords: Liraglutide; Type 2 diabetes; Elderly; A1C; Weight; Severe hypoglycemia; Clinical effectiveness

\section{Introduction}

In recent years, diabetes mellitus has emerged as a global public health concern. It is the most common metabolic disorder and the global prevalence rates have been increasing [1]. Among the United States (U.S.) population ages 65 years and older, 26.9 percent $(10.9$ million) had diabetes in 2010, which was the seventh leading cause of death in the U.S. [1]. The American Diabetes Association (ADA) estimated that healthcare costs for the $\geq 65$ year old population totaled $\$ 104$ billion in 2012, which represents about 59\% of total diabetesrelated direct healthcare costs. This population utilized a substantially higher proportion of healthcare services such as hospital inpatient days, nursing/residential facility days, and hospice care compared with those below age 65 years [2]. Many complications linked to diabetes can be more prevalent, and more costly, in this population, including hypertension, myocardial infarction, stroke, other heart diseases, kidney disease, and several others, which could potentially incur further costs [1].

Liraglutide is a once daily injectable glucagon-like peptide-1 (GLP1) analogue that was approved by the FDA in 2010. Liraglutide is indicated as an adjunct to diet and exercise to improve glycemic control in adults with type 2 diabetes (T2D) [3]. The pharmacokinetic profile of liraglutide makes it suitable for once daily administration, which assists in glucose normalization in T2D patients throughout the day [3-5]. Liraglutide users are started at $0.6 \mathrm{mg}$ per day for one week and then the dose is increased to $1.2 \mathrm{mg}$ and can then be increased to $1.8 \mathrm{mg}$ if glycemic control is inadequate [3].
The phase III clinical trial program for liraglutide, known as LEAD (Liraglutide Effect and Action in Diabetes), included different treatment regimens as active comparators [6-8]. These studies have demonstrated liraglutide's efficacy to safely lower A1C and body weight, while minimizing the risk of minor and severe hypoglycemia. Although these studies contribute to scientific knowledge of diabetes treatments overall, they focused on subjects whose mean ages ranged from 53 to 57 years, which may have limited the ability of those studies to fully characterize the impact of treatment in individuals aged 65 years and older [8-14].

Individuals 65 years and older may have additional age-related health complications that can affect the treatment outcomes. A review of studies of T2D treatments that examined efficacy and tolerability in individuals 65 years and older found that treatment-specific benefitrisk trade-offs were different for individuals below 65 years than for those above 65 years [15]. Specifically, one study comparing the groups found individuals in this age to have a greater mean A1C reduction with a similar reporting of hypoglycemia while on a dipeptidyl peptidase- 4

*Corresponding author: Abhishek Chitnis, Senior Research Associate, Retrospective Observational Studies, Evidera 430 Bedford Street, Suite 300, Lexington, MA 02420, USA, Tel: +1 781-960-0256; E-mail: abhishek.chitnis@evidera.com

Received June 06, 2014; Accepted June 27, 2014; Published July 04, 2014

Citation: Chitnis A, Ganz ML, Hammer M, Langer J (2014) Real-World Clinical Effectiveness of Liraglutide in Individuals 65 Years and Older with Type 2 Diabetes in the United States. J Diabetes Metab 5: 403 doi:10.4172/2155-6156.1000403

Copyright: $\odot 2014$ Chitnis A, et al. This is an open-access article distributed under the terms of the Creative Commons Attribution License, which permits unrestricted use, distribution, and reproduction in any medium, provided the original author and source are credited. 
(DPP-4) inhibitor [15]. However, a pooled analysis of patients from the LEAD studies that compared the efficacy of liraglutide by age group cohorts $(<65$ and $\geq 65)$ found liraglutide $1.2 \mathrm{mg}$ and liraglutide $1.8 \mathrm{mg}$ to be equally efficacious in individuals $\geq 65$ years as in individuals $<65$ years [6].

While several studies have demonstrated that liraglutide can lower A1C levels and reduce weight in T2D, data are still very scarce on its use in individuals 65 years and older, particularly in clinical practice. Clinical guidance on the treatment of T2D is largely based on data observed from a younger sample, which may not adequately translate into an older population. Moreover, these results are mainly from clinical trials and need to be confirmed using real-world clinical practice outcomes. Patient follow-up time reported in previous studies was mostly limited to 6 months, which may not be sufficient to accurately demonstrate long term effects $[10,12,13,16-20]$.

The aim of this study was to address these data gaps by evaluating the effectiveness of liraglutide at 6 and 12 months after initiating therapy in individuals 65 years and older with T2D.

\section{Methods}

\section{Data source}

This was a retrospective cohort based study using the General Electric (GE) Centricity Electronic Medical Record (EMR) dataset from January 1, 2009, to January 31, 2013. The GE Centricity EMR database contains data on more than 15 million individuals who receive care from more than 10,000 general practitioners. Forty-seven US states are represented and the average length of follow-up for individuals in the dataset is approximately 3 years. This database includes detailed information regarding patient demographic characteristics that are not available in a typical medical claims database, such as patient height, weight, Body Mass Index (BMI), and smoking status. The GE Centricity EMR database also includes laboratory results not available in most claims databases such as cholesterol, blood glucose, A1C. This general practitioner (ambulatory) EMR database provides complete information on all prescribed drugs for patients receiving care from that practice. Patient information from a variety of sources is routinely integrated into a common database and includes number of patient encounters, insurance data, medication data that reflect not only prescription drug data, but also over-the-counter (OTC) medications prescribed by the physician and historical drug use. This study was exempt from ethics approval from an institutional review board and informed consent since it involved assessment of existing data, and the subjects could not be identified directly or through identifiers linked to the subjects (45 CFR 46.101(b)).

\section{Sample selection}

Individuals were included in the study sample if they had T2D and had a prescription written for liraglutide between January 1, 2010, and January 31,2013. The index date was defined as the date of the first prescription for liraglutide. T2D was defined as a patient who met one of the following criteria: (i) At least one diagnosis for T2D based on an International Classification of Diseases, Ninth Revision, Clinical Modification (ICD-9-CM) code for 250.x0 or 250.x2; (ii) One or more prescription orders for a non-insulin antidiabetic drug; or (iii) Two consecutive fasting blood glucose levels of $\geq 126 \mathrm{mg} / \mathrm{d}$ [21]. Our analysis focused on the outcome measures at two follow-up periods: 6 and 12 months post-index date (Figure 1).

Individuals were excluded if they (i) Were not continuously enrolled during 12 months prior to the index date (pre-index period)

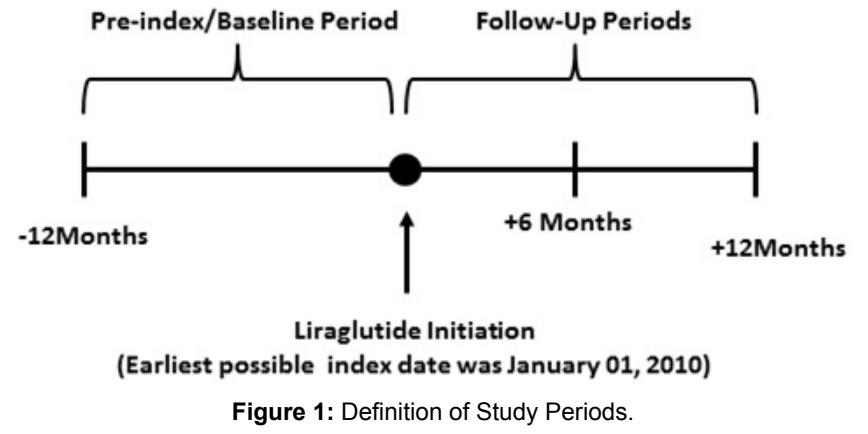

and during 6 and 12 months follow-up; (ii) Were less than 65 years old at the index date; (iii) Had one or more prescription orders for any GLP1 during baseline; (iv) Had one or more prescription orders for insulin use during pre-index period; (v) Had any of the following conditions during the pre-index period, type 1 diabetes (ICD-9-CM codes: 250. $\mathrm{x} 1$ or $250 . \mathrm{x} 3$ ), polycystic ovarian syndrome (ICD-9-CM code 256.4) without the presence of T2D (ICD-9-CM codes $250 . x 0$ or 250.x2); (vi) Individuals already 'in control' who had a baseline A1C $\leq 7 \%$ [22]. All patients were required to have at least one valid A1C measure at baseline (up to 45 days prior to the index date to up to 7 days after). For follow-up periods ( 6 and 12 months) the patients were required to have at least one valid A1C measure at follow-up (up to 45 days before or after 180 and 360 days, respectively) in addition to at least one valid $\mathrm{A} 1 \mathrm{C}$ measure at baseline.

\section{Demographic and clinical characteristics}

Demographic characteristics like age, sex, race, geographic region (Midwest, Northeast, South, and West), and health plan type (Commercial, Medicare, Medicaid, Self-pay/Other, and Unknown) were assessed at baseline. Baseline clinical characteristics included BMI and common diabetes-related comorbidities [23], identified using ICD9-CM codes (Appendix A). Clinical measures such as A1C, weight, blood pressure (systolic blood pressure, SBP; diastolic blood pressure, DBP) lipid values (total cholesterol; high density lipoprotein cholesterol, HDL) and occurrence of severe hypoglycemia were reported at both the baseline and follow-up periods ( 6 and 12 months). Severe hypoglycemia was defined according to type of service and ICD-9-CM codes and/or blood glucose levels; (i) For outpatient or inpatient services: ICD-9CM codes 251.0x, 251.1x, 251.2x, and 250.3x on different days; (ii) For emergency department visits: ICD-9-CM codes $251.8 \mathrm{x}$ without $259.8 \mathrm{x}$, 272.7, 681.xx, 682.xx, 686.9x, 707.1x-707.9x, 709.3x, 730.0x-730.2x, and $731.8 \mathrm{x}$ ); (iii) A recorded glucose level of less than or equal to 40 $\mathrm{mg} / \mathrm{dL}[24,25]$.

Patients may have had multiple measurements of the clinical outcomes during the baseline and follow-up periods. The baseline value was defined as the value that was closest to the index date ("day 0 ") that was within a window of between 45 days before to 7 days after the index date. The 6-month value was the measurement obtained on the day closest to day 180 within a \pm 45 -day window and the 12 month value was the measurement obtained on the day closest to day 360 within a \pm 45 day window.

\section{Clinical outcomes}

We assessed the following clinical outcomes at 6 and 12 months: absolute changes (follow-up minus baseline values) in $\mathrm{A} 1 \mathrm{C}$, body weight, blood pressure, and lipids, relative changes (absolute change divided by baseline values) in body weight, and the proportion of 
liraglutide users reaching A1C targets. We used both the American Association of Clinical Endocrinologists (AACE) target of A1C $\leq 6.5 \%$ and the American Diabetes Association (ADA) target of $\mathrm{A} 1 \mathrm{C}<7 \%$. A relaxed target of $\mathrm{A} 1 \mathrm{C}<7.5 \%$ as suggested by the ADA Consensus Development Conference on Diabetes and Older Adults was used as well [26]. Finally, we examined the occurrence of severe hypoglycemia at 6 and 12 months.

\begin{tabular}{|c|c|}
\hline Characteristic & Estimate \\
\hline Age & $70.6(4.7)$ \\
\hline \multicolumn{2}{|l|}{ Sex, \% } \\
\hline Female & 52.6 \\
\hline Male & 47.4 \\
\hline \multicolumn{2}{|l|}{ Race/ethnicity, \% } \\
\hline Caucasian & 71.6 \\
\hline African American & 4.1 \\
\hline Hispanic & 0.2 \\
\hline Other & 2.5 \\
\hline Unknown & 21.7 \\
\hline \multicolumn{2}{|l|}{ Region, \% } \\
\hline Midwest & 21.1 \\
\hline Northeast & 21.1 \\
\hline South & 43.7 \\
\hline West & 14.1 \\
\hline \multicolumn{2}{|l|}{ Plan type, $\%$} \\
\hline Commercial & 10.6 \\
\hline Medicare & 54.7 \\
\hline Medicaid & 0 \\
\hline Self-pay/other & 0.4 \\
\hline Unknown & 34.2 \\
\hline $\mathrm{BMI}, \mathrm{kg} / \mathrm{m}^{2}$ & $35.8(6.5)$ \\
\hline \multicolumn{2}{|l|}{ BMI categories, $\%$} \\
\hline $25.0-29.9 \mathrm{~kg} / \mathrm{m}^{2}$ & 16.8 \\
\hline $30.0-34.9 \mathrm{~kg} / \mathrm{m}^{2}$ & 33.7 \\
\hline $35.0-39.9 \mathrm{~kg} / \mathrm{m}^{2}$ & 28.1 \\
\hline$\geq 40.0 \mathrm{~kg} / \mathrm{m}^{2}$ & 21.5 \\
\hline \multicolumn{2}{|l|}{ Comorbid conditions, $\%$} \\
\hline Retinopathy & 0.2 \\
\hline Nephropathy & 3.7 \\
\hline Neuropathy & 1.7 \\
\hline Cerebrovascular & 0.4 \\
\hline Cardiovascular & 1.7 \\
\hline Peripheral vascular disease & 0.2 \\
\hline Metabolic & 0 \\
\hline \multicolumn{2}{|l|}{ Smoking status, $\%$} \\
\hline Current smoker & 3.3 \\
\hline Former smoker & 37.5 \\
\hline Never smoked & 21.7 \\
\hline Other/unknown & 37.5 \\
\hline A1C, $\%$ & $8.22(0.97)$ \\
\hline Weight, kg & $101.0(19.7)$ \\
\hline \multicolumn{2}{|l|}{ Lipids mg/dl } \\
\hline Total cholesterol & $166.6(44.1)$ \\
\hline HDL & $43.6(12.6)$ \\
\hline \multicolumn{2}{|l|}{ Blood pressure $\mathrm{mmHg}$} \\
\hline SBP & $131.9(15.4)$ \\
\hline DBP & $73.9(9.1)$ \\
\hline
\end{tabular}

Table 1: Baseline Characteristics of Study Sample $(\mathrm{N}=517)$.

\section{Analyses}

Means and standard deviations (SD) were reported for continuous measures, and percentages were reported for categorical measures. Statistical significance between baseline and follow-up values were assessed using the paired t-test for continuous measures and McNemar's test for categorical measures. Differences with a p-value of less than 0.05 were considered statistically significant. Analyses were performed using SAS software version 9.2 (SAS Institute, Cary, NC).

\section{Results}

\section{Baseline characteristics}

We identified and included 517 individuals with T2D who were 65 years or older with uncontrolled blood glucose (A1C $>7 \%)$ at baseline and who had a prescription order for liraglutide between January 1 , 2010, and January 31, 2013. Table 1 shows their baseline demographic and clinical characteristics. The mean age (SD) of the study sample was 70.6 (4.7) years. About 53\% were female and $72 \%$ were white. About $44 \%$ resided in the south and $55 \%$ were covered by a Medicare health plan. The most common comorbid condition was nephropathy (4\%) followed by cardiovascular disorder (2\%) and neuropathy (2\%). The mean BMI (SD) in the sample was $35.8 \mathrm{~kg} / \mathrm{m}^{2}(6.5)$; proportionately more patients were in the $30.0-34.9 \mathrm{~kg} / \mathrm{m}^{2}$ BMI category $(33.7 \%)$ compared with other BMI categories. At baseline, the mean A1C was $8.22 \%(0.97 \%)$ and the average weight was 101.0 (19.7) $\mathrm{kg}$ [222.2 (43.3) lbs]. SBP and DBP were, on average, 131.9 (15.4) $\mathrm{mmHg}$ and 73.9 (9.1) $\mathrm{mmHg}$, respectively and average total cholesterol and HDL were 166.6 (44.1) $\mathrm{mg} / \mathrm{dl}$ and 43.6 (12.6) $\mathrm{mg} / \mathrm{dl}$, respectively (Table 1). No severe hypoglycemic events were identified during the baseline period.

\section{Clinical outcomes}

Of 517 individuals treated with liraglutide, 313 had A1C measures at both baseline and 6 months follow-up, whereas 218 individuals had A1C measures at baseline and 12 months follow-up. Tables 2 and 3 show the clinical outcomes for liraglutide at 6 and 12 months follow-up. Average A1C values at 6 and 12 months of follow-up were 7.45\% (1.22\%) and $7.41 \%(1.22 \%)$, respectively. There were significant decreases in the mean $\mathrm{A} 1 \mathrm{C}$ after 6 and 12 months of follow-up. The mean absolute change in A1C at 6 and 12 months of follow-up were $-0.76 \%$ (1.14\%) and $-0.78 \%(1.16 \%)$ (both $\mathrm{P}<0.01)$ compared with baseline respectively. At 6 months $22.0 \%, 37.1 \%$, and $58.5 \%$ of individuals achieved A1C $\leq 6.5 \%,<7 \%$ and $<7.5 \%$ targets, respectively. Similarly, at 12 months $23.4 \%, 39.9 \%$ and $59.6 \%$ of individuals achieved $\mathrm{A} 1 \mathrm{C} \leq 6.5 \%,<7 \%$ and $<7.5 \%$ targets, respectively.

Average body weight at 6 and 12 months of follow-up were 97.8 (18.6) $\mathrm{kg}$ [215.2 (40.9) lbs] and $98.2(18.6) \mathrm{kg}$ [216.0 (40.9) lbs], respectively. At 6 months follow-up, the absolute and relative changes (SD) in body weight were -2.9 (5.4) $\mathrm{kg}$ [6.4 (11.9) lbs] and $-3.0 \%(1.0 \%)$ respectively. Twelve-month changes were similar at $-3.1 \mathrm{~kg}(5.7)$ [6.8 (12.5) lbs] and $-3.0 \%(1.0 \%)$. Both the absolute and relative changes in body weight at 6 and 12 months of follow-up were statistically significantly different from zero $(\mathrm{P}<0.01)$. Statistically significant reductions in total cholesterol values were also observed at both follow-up periods $(\mathrm{P}<0.01)$. The HDL levels, however, were similar between baseline and follow-up periods. SBP changed by $-2.9(17.4) \mathrm{mmHg}(\mathrm{P}<0.01)$ at 6 months and by $-2.1(17.2)$ $\mathrm{mmHg}(\mathrm{P}=0.08)$ at 12 months. $\mathrm{DBP}$ changed by $-0.8(9.6) \mathrm{mmHg}(\mathrm{P}=0.17)$ and by $-1.4(9.4) \mathrm{mmHg}(\mathrm{P}=0.03)$. No severe hypoglycemic episodes were identified among liraglutide patients at 6 or 12 months follow-up. There were no notable differences between comorbid conditions at both followup periods as compared to baseline (data not shown here). 


\begin{tabular}{|c|c|c|c|c|}
\hline Clinical Measure & Baseline & 6 Months & Change* & $\mathbf{P}$ \\
\hline$A 1 C$, mean (SD) $(\mathrm{N}=313)$ & $8.22(0.97)$ & $7.45(1.22)$ & $-0.76(1.14)$ & $<0.01$ \\
\hline \multicolumn{5}{|l|}{$\mathrm{A} 1 \mathrm{C}, \%(\mathrm{~N}=313)$} \\
\hline$\leq 6.5 \% \%^{* *}$ & & 22 & & \\
\hline$<7.0 \%$ ** & & 37.1 & & \\
\hline$<7.5 \%$ & 19.8 & 58.5 & 38.7 & $<0.01$ \\
\hline \multirow[t]{2}{*}{ Weight $(\mathrm{kg})$, mean (SD) (N=299) } & $100.7(19.0)$ & $97.8(18.6)$ & $-2.9(5.4)$ & $<0.01$ \\
\hline & & & $-3.0 \%(1.0)^{\star * *}$ & $<0.01$ \\
\hline \multicolumn{5}{|l|}{ Lipids mg/dl } \\
\hline Total Cholesterol, mean (SD) $(\mathrm{N}=148)$ & $166.2(42.3)$ & $156.5(39.2)$ & $-9.6(38.4)$ & $<0.01$ \\
\hline HDL, mean (SD) $(\mathrm{N}=145)$ & $42.8(11.8)$ & $42.8(12.6)$ & $0.04(6.2)$ & 0.94 \\
\hline \multicolumn{5}{|l|}{ Blood pressure $\mathrm{mmHg}(\mathrm{N}=297)$} \\
\hline SBP, mean (SD) & $132.0(16.0)$ & $129.2(15.4)$ & $-2.9(17.4)$ & $<0.01$ \\
\hline DBP, mean (SD) & $73.4(8.9)$ & $72.6(9.7)$ & $-0.8(9.6)$ & 0.17 \\
\hline
\end{tabular}

Table 2: Liraglutide clinical measures at baseline and 6 months, ${ }^{*}$ Changes shown are absolute (6 month value minus baseline value) unless otherwise noted. **Patients were included in the study only if baseline $\mathrm{A} 1 \mathrm{C}$ was greater than $7 \%$. ${ }^{* *}$ Relative change (=absolute change divided by baseline value). Note: Data expressed as mean (standard deviation) unless otherwise noted. HDL, high density lipoprotein cholesterol; SBP, systolic blood pressure; DBP, diastolic blood pressure.

\begin{tabular}{|c|c|c|c|c|}
\hline Clinical Measure & Baseline & 12 Months & Change* $^{*}$ & $\mathbf{P}$ \\
\hline A1C, mean (SD) $(\mathrm{N}=218)$ & $8.19(0.94)$ & $7.41(1.22)$ & $-0.78(1.16)$ & $<0.01$ \\
\hline \multicolumn{5}{|l|}{$\mathrm{A} 1 \mathrm{C}, \%(\mathrm{~N}=218)$} \\
\hline$\leq 6.5 \%{ }^{* *}$ & & 23.4 & & \\
\hline$<7.0 \%{ }^{* *}$ & & 39.9 & & \\
\hline$<7.5 \%$ & 20.6 & 59.6 & 39.0 & $<0.01$ \\
\hline \multirow[t]{2}{*}{ Weight $(\mathbf{k g})$, mean $(\mathrm{SD})(\mathrm{N}=209)$} & $101.3(18.8)$ & $98.2(18.6)$ & $-3.1(5.7)$ & $<0.01$ \\
\hline & & & $-3.0 \%(1.0)^{\star * *}$ & $<0.01$ \\
\hline \multicolumn{5}{|l|}{ Lipids mg/dl } \\
\hline Total Cholesterol mean (SD) $(\mathrm{N}=115)$ & $168.9(41.1)$ & $154.2(30.8)$ & $-10.7(35.0)$ & $<0.01$ \\
\hline $\mathrm{HDL}$, mean (SD) $(\mathrm{N}=112)$ & $42.4(11.1)$ & $43.0(10.9)$ & $0.6(5.8)$ & 0.25 \\
\hline \multicolumn{5}{|l|}{ Blood pressure $\mathrm{mmHg}(\mathrm{N}=\mathbf{2 0 8})$} \\
\hline SBP, mean (SD) & $131.2(14.8)$ & $129.1(13.3)$ & $-2.1(17.2)$ & 0.08 \\
\hline DBP, mean (SD) & $73.7(8.8)$ & $72.3(9.2)$ & $-1.4(9.4)$ & 0.03 \\
\hline
\end{tabular}

Table 3: Liraglutide clinical measures at baseline and 12 months; ${ }^{*}$ Changes shown are absolute (12-month value minus baseline value) unless otherwise noted. ** Patients were included in the study only if baseline $\mathrm{A} 1 \mathrm{C}$ was greater than $7 \%$. ${ }^{* *}$ Relative change (=absolute change divided by baseline value). Note: Data expressed as mean (standard deviation) unless otherwise noted. HDL, high density lipoprotein cholesterol; SBP, systolic blood pressure; DBP, diastolic blood pressure.

\section{Discussion}

This study contributes to the existing literature by providing realworld evidence of the effectiveness of liraglutide for individuals with T2D who are 65 years or older. To the best of our knowledge, this is the first study to examine the effectiveness of liraglutide for individuals 65 years and older based on clinical practice data. We found that initiating liraglutide was associated with significant reductions in A1C and weight at 6 months of follow-up. Similar significant associations were observed in $\mathrm{A} 1 \mathrm{C}$ and weight over the longer term period of 12 months after initiating liraglutide, signifying a sustained treatment effect. Furthermore, no cases of severe hypoglycemia were observed at 6 and 12 months follow-up. Focusing on this age group is important for several reasons. First, most existing evidence for liraglutide comes from randomized, phase 3 trials that enrolled younger subjects (average age of 56 years), which may not generalize to older people with T2D. Second, a growing body of evidence shows that individuals 65 years and older with T2D may face more challenges than younger individuals, often because of comorbid conditions that may lead to polypharmacy and an increased risk of drug-disease and drug-drug interactions that may limit the range of antidiabetic agents that can be used [6,27]. Third, as pointed about above, is that there is no published evidence around the real-world effectiveness of liraglutide for individuals 65 years and older.

Focusing on this age group is also important because elderly individuals with T2D are at higher risk for severe hypoglycemia than younger individuals $[28,29]$. The clinical burden of severe hypoglycemia, cardiovascular events, cognitive impairment and fractures due to falls in the elderly is greater than in younger patients [6]. Our study found no evidence of severe hypoglycemia either after 6 or at 12 months follow-up.

Bode et al. pooled and analyzed six randomized, placebocontrolled, multinational trials to assess the efficacy and tolerability profile of liraglutide in individuals $\geq 65$ years and $<65$ years with T2D at 26 weeks [6]. They found statistically significant differences in A1C change in both groups from baseline. The mean reduction in $\mathrm{AlC}$ in individuals $\geq 65$ years at 26 weeks was $1.34 \%$ and about $61 \%$ achieved the ADA target of A1C $<7 \%$. We found a slightly lower reduction in the $\mathrm{A} 1 \mathrm{C}(0.76 \%)$ and in the proportion of individuals meeting target of 
$\mathrm{A} 1 \mathrm{C}<7 \%(41.2 \%)$ at 6 months. This could be due to differences between the strictly controlled clinical trial setting and real-world impacts of physician practice patterns, patient adherence to therapy, and the use and presence of concomitant medications and comorbid conditions.

The pooled analysis of clinical trials showed statistically significant differences in weight reduction between individuals with T2D in the $\geq$ 65 and $<65$ year age groups at 26 weeks. They reported that individuals $\geq 65$ years lost on average $1.6 \mathrm{~kg}[3.5 \mathrm{lbs}]$ at 26 weeks whereas we found that our sample lost on average $2.9 \mathrm{~kg}$ [6.4 lbs] (3.0\% relative change; Bode et al. did not report relative change) at 6 month follow-up. This may be due to the differences in baseline characteristics of the patients in the trials and in our sample. The individuals in our study had a higher average baseline BMI $\left(35.8 \mathrm{~kg} / \mathrm{m}^{2}\right)$ than those in the pooled analysis $\left(30.6 \mathrm{~kg} / \mathrm{m}^{2}\right)$. Our study included about $50 \%$ of individuals with BMI $\geq$ $35.0 \mathrm{~kg} / \mathrm{m}^{2}$ and the findings are consistent with those trials that enrolled participants with a higher baseline BMI [18,30,31]. Evans et al. [32] conducted a retrospective chart audit of individuals with mean baseline BMI of $39.5 \mathrm{~kg} / \mathrm{m}^{2}$ receiving liraglutide and reported similar weight loss of $2.9 \mathrm{~kg}$ [6.4 lbs] 6 months after starting liraglutide.

These results are consistent with the pooled meta-analysis from the LEAD trials that found patients 65 years and older did not experience any major hypoglycemic events over 26 weeks [6]. This could be due to liraglutide's glucose dependent mechanism of action; whereas certain other anti-diabetic treatments stimulate insulin secretion regardless of the blood glucose level causing severe hypoglycemia.

Furthermore, there are limited trial data available on the long term effects ( $\geq 1$ year) of liraglutide in T2D patients and none of these studies have assessed the effects in patients 65 years and older [16,33,34]. Garber et al found that A1C decreased by $0.84 \%$ for liraglutide $1.2 \mathrm{mg}$ and $1.14 \%$ for liraglutide $1.8 \mathrm{mg}$ at 52 weeks [16]. Further, when these individuals were followed for an additional year the A1C reduction was $0.90 \%$ and $1.10 \%$. Pratley et al. reported mean A1C reductions from baseline to week 52 of $1.29 \%$ with $1.2 \mathrm{mg}$ and $1.51 \%$ with $1.8 \mathrm{mg}$ liraglutide [34]. The decreases in $\mathrm{A} 1 \mathrm{C}$ and the proportion of patients achieving the $\mathrm{ADA} \mathrm{AlC}<7.0 \%$ target from the trial data were slightly higher than with our $\mathrm{A} 1 \mathrm{C}$ reduction of $0.78 \%$ and proportion of patients achieving A1C $<7.0 \%$ target of $39.9 \%$ at 12 months. This could be due to differences in the age of the study populations or trial design. The mean age of patients recruited in these trials was about 54 years, which is much younger than our study cohort (70.6 years). However, the weight loss finding was consistent with the one-year trial data. Garber et al found that the mean weight change at 52 weeks with liraglutide $1.2 \mathrm{mg}$ was $-2.6 \mathrm{~kg}[-5.7 \mathrm{lbs}]$ and with liraglutide $1.8 \mathrm{mg}$ was $-2.9 \mathrm{~kg}$ [-6.4 lbs] [16]. Our study found an absolute change in weight of about $-3.1 \mathrm{~kg}$ [-6.8 lbs] (-3.0\% relative change).

Hypertension is a known risk factor for cardiovascular disease, diabetic nephropathy and diabetic retinopathy [8]. SBP reductions have been shown to reduce the relative risk of death from cardiovascular disease and death from any cause [8]. The mean decreases in systolic and diastolic blood pressure found in our study were $2.9 \mathrm{mmHg}$ and $0.8 \mathrm{mmHg}$ at 6 months and $2.1 \mathrm{mmHg}$ and $1.4 \mathrm{mmHg}$ at 12 months respectively. These results are consistent with the trial data [6]. A retrospective real-world study by Mulligan et al. [11] found a similar change in SBP in a cohort of patients in a hospital setting taking liraglutide. However, the study only looked at the short term effects from baseline to first clinic visit after initiating liraglutide with an average time of 13.5 weeks for the first clinic visit. A retrospective chart audit found similar decrease in blood pressure, $2.4 \mathrm{mmHg}$ at 6 months and $2.2 \mathrm{mmHg}$ at 12 months, with liraglutide treatment. However, the study failed to distinguish between SBP and DBP. The weight loss reported by Evans et al. in liraglutide individuals was also consistent with our study at both 6 and 12 months [32]. Furthermore, we found statistically significant decreases in total cholesterol levels at 6 and 12 months. The HDL levels observed at 6 and 12 months were similar to those at baseline. This result is similar to the trial data in which no statistical significant change in HDL was observed; however there was a small increase in numerical value [34]. Overall, the observed changes in blood pressure and lipids may be due to the direct or indirect effects of liraglutide treatment (e.g. weight loss).

There are a number of limitations of this study. The study measures of interest were not available for all individuals at all time periods. Identifying laboratory and clinical values at both baseline and followup windows for the study endpoints resulted in sample attrition over the follow-up time periods. A natural limitation of applying pre-defined baseline and follow-up windows in retrospective database analyses, to assess changes in clinical outcomes, is that these values may not reflect and capture the complete clinical information of the identified subjects over the entire follow-up period. Because the GE Centricity data are limited to general practice ambulatory care settings, certain encounters, such as specialist care and hospitalizations, as well as complications experienced in those settings, were not captured, possibly resulting in an undercount of hypoglycemic events. We were also not able to capture minor hypoglycemic events and other most common adverse events have been described in the trials [20]. The information on duration of disease was not complete for all patients and thus not captured. As it is not possible to ascertain if people actually filled or refilled their prescriptions using this database, adherence to therapy was not examined and, therefore, the study used an intent-to-treat approach. The information on dose was often missing, which is the reason why we did not stratify any analyses by liraglutide dose. Medication start and end dates were recorded by the physician, which may not reflect actual medication utilization. Despite these limitations, our study adds to the evidence base of the effectiveness of liraglutide at 6 and 12 months follow-up in real-world clinical practice in individuals 65 years and older with T2D. Our study confirms the efficacy data from the LEAD trials by demonstrating significant and sustained reductions in A1C and weight at 6 and 12 months after initiating liraglutide. Moreover, our study finding of no severe hypoglycemia 6 and 12 months followup after initiating liraglutide confirms the tolerability data from the LEAD trials. The results from this study could be used by clinicians and patients in the decision making for treatment options in people 65 years or older with T2D.

\section{Conflict of Interest and Financial Support}

AC, MG are employees of Evidera, which provides consulting and other research services to pharmaceutical, device, government, and non-government organizations. In this salaried position, they work with a variety of companies and organizations and are precluded from receiving payment or honoraria directly from these organizations for services rendered. JL and MH are employees of Novo Nordisk and are shareholders of Novo Nordisk.

\section{Author Contributions}

AC made signification contributions to the analysis and interpretation of the data and wrote a significant portion of the manuscript. MG participated in the study design and critically reviewed and edited the manuscript. JL and MH contributed to the study conception and design and reviewed the manuscript. All authors read and approved the final manuscript. 
Citation: Chitnis A, Ganz ML, Hammer M, Langer J (2014) Real-World Clinical Effectiveness of Liraglutide in Individuals 65 Years and Older with Type 2 Diabetes in the United States. J Diabetes Metab 5: 403 doi:10.4172/2155-6156.1000403

Page 6 of 6

\section{References}

1. Barnes PM, Schoenborn CA (2012) Trends in adults receiving a recommendation for exercise or other physical activity from a physician or other health professional. NCHS Data Brief : 1-8.

2. American Diabetes Association1 (2013) Economic costs of diabetes in the U.S in 2012. Diabetes Care 36: 1033-1046.

3. Novo Nordisk.Victoza (liraglutide injection) prescribing information. In: A/S NN ed2013.

4. Cho YM, Wideman RD2, Kieffer TJ2 (2013) Clinical application of glucagonlike Peptide 1 receptor agonists for the treatment of type 2 diabetes mellitus. Endocrinol Metab (Seoul) 28: 262-274

5. Perry CM (2011) Liraglutide: a review of its use in the management of type 2 diabetes mellitus. Drugs 71: 2347-2373.

6. Bode BW, Brett J, Falahati A, Pratley RE (2011) Comparison of the efficacy and tolerability profile of liraglutide, a once-daily human GLP-1 analog, in patients with type 2 diabetes 65 and $<65$ years of age: a pooled analysis from phase III studies. Am J Geriatr Pharmacother 9: 423-433.

7. Bailey T (2013) Options for combination therapy in type 2 diabetes: comparison of the ADA/EASD position statement and AACE/ACE algorithm. Am J Med 126 S10-20.

8. Garber AJ (2012) Liraglutide in oral antidiabetic drug combination therapy Diabetes Obes Metab 14 Suppl 2: 13-19.

9. Fadini GP, Simioni N, Frison V, Dal Pos M, Bettio M, et al. (2013) Independent glucose and weight-reducing effects of Liraglutide in a real-world population of type 2 diabetic outpatients. Acta Diabetol 50: 943-949.

10. Kesavadev J, Shankar A, Krishnan G, Jothydev S (2012) Liraglutide therapy beyond glycemic control: an observational study in Indian patients with type 2 diabetes in real world setting. Int J Gen Med 5: 317-322.

11. Mulligan CM, Harper R, Harding J, Mcllwaine W, Petruckevitch A, et al. (2013) A retrospective audit of type 2 diabetes patients prescribed liraglutide in real-life clinical practice. Diabetes Ther 4: 147-151.

12. Zinman B, Schmidt WE, Moses A, Lund N, Gough S (2012) Achieving clinically relevant composite outcome of an $\mathrm{HbA} 1 \mathrm{c}$ of $<7 \%$ without weight gain or hypoglycaemia in type 2 diabetes: a meta-analysis of the liraglutide clinical trial programme. Diabetes Obes Metab 14:77-82.

13. DeKoven M, Lee WC, Bouchard J, Massoudi M, Langer J (2014) Realworld cost-effectiveness: lower cost of treating patients to glycemic goal with liraglutide versus exenatide. Adv Ther 31: 202-216.

14. Langer J, Hunt B, Valentine WJ (2013) Evaluating the short-term costeffectiveness of liraglutide versus sitagliptin in patients with type 2 diabetes failing metformin monotherapy in the United States. J Manag Care Pharm 19 237-246.

15. Germino FW (2011) Noninsulin treatment of type 2 diabetes mellitus in geriatric patients: a review. Clin Ther 33: 1868-1882.

16. Garber A, Henry R, Ratner R, Garcia-Hernandez PA, Rodriguez-Pattzi H, et al (2009) Liraglutide versus glimepiride monotherapy for type 2 diabetes (LEAD-3 Mono): a randomised, 52-week, phase III, double-blind, parallel-treatment trial. Lancet 373: 473-481.

17. Marre M, Shaw J, Brändle M, Bebakar WM, Kamaruddin NA, et al. (2009) Liraglutide, a once-daily human GLP-1 analogue, added to a sulphonylurea over 26 weeks produces greater improvements in glycaemic and weight control compared with adding rosiglitazone or placebo in subjects with Type 2 diabetes (LEAD-1 SU). Diabet Med 26: 268-278.

18. Buse JB, Rosenstock J, Sesti G, Schmidt WE, Montanya E, et al. (2009) Liraglutide once a day versus exenatide twice a day for type 2 diabetes: a 26-week randomised, parallel-group, multinational, open-label trial (LEAD-6). Lancet 374: 39-47.

19. Nauck M, Frid A, Hermansen K, Shah NS, Tankova T, et al. (2009) Efficacy and safety comparison of liraglutide, glimepiride, and placebo, all in combination with metformin, in type 2 diabetes: the LEAD (liraglutide effect and action in diabetes)-2 study. Diabetes Care 32: 84-90.

20. Pratley RE, Nauck M, Bailey T, Montanya E, Cuddihy R, et al. (2010) Liraglutide versus sitagliptin for patients with type 2 diabetes who did not have adequate glycaemic control with metformin: a 26-week, randomised, parallel-group, open-label trial. Lancet 375: 1447-1456.

21. Brixner DI, McAdam-Marx C, Ye X, Boye KS, Nielsen LL, et al. (2009) Sixmonth outcomes on $\mathrm{A} 1 \mathrm{C}$ and cardiovascular risk factors in patients with type 2 diabetes treated with exenatide in an ambulatory care setting. Diabetes Obes Metab 11:1122-1130.

22. American Diabetes Association (2013) Standards of medical care in diabetes-2013. Diabetes Care 36 Suppl 1: S11-66.

23. Young BA, Lin E, Von Korff M, Simon G, Ciechanowski P, et al. (2008) Diabetes complications severity index and risk of mortality, hospitalization, and healthcare utilization. Am J Manag Care 14: 15-23.

24. Ginde AA, Blanc PG, Lieberman RM, Camargo CA Jr (2008) Validation of ICD9-CM coding algorithm for improved identification of hypoglycemia visits. BMC Endocr Disord 8: 4 .

25. Elliott MB, Schafers SJ, McGill JB, Tobin GS (2012) Prediction and prevention of treatment-related inpatient hypoglycemia. J Diabetes Sci Technol 6: 302-309.

26. Sue Kirkman M, Briscoe VJ, Clark N, Florez H, Haas LB, et al. (2012) Diabetes in older adults: a consensus report. J Am Geriatr Soc 60: 2342-2356.

27. Neumiller JJ, Setter SM (2009) Pharmacologic management of the olde patient with type 2 diabetes mellitus. Am J Geriatr Pharmacother 7: 324-342.

28. Chan TY (1998) Estimates on the incidence of antidiabetic drug-induced severe hypoglycaemia in Hong Kong. Pharmacoepidemiol Drug Saf 7: 411-414.

29. Holstein A, Plaschke A, Egberts EH (2003) Clinical characterisation of severe hypoglycaemia-a prospective population-based study. Exp Clin Endocrino Diabetes 111: 364-369.

30. Buse JB, Nauck M, Forst T, Sheu WH, Shenouda SK, et al. (2013) Exenatide once weekly versus liraglutide once daily in patients with type 2 diabetes (DURATION-6): a randomised, open-label study. Lancet 381: 117-124.

31. Niswender K, Pi-Sunyer X, Buse J, Jensen KH, Toft AD, et al. (2013) Weigh change with liraglutide and comparator therapies: an analysis of seven phase 3 trials from the liraglutide diabetes development programme. Diabetes Obes Metab 15: 42-54.

32. Evans M, McEwan P, O'Shea R, George L (2013) A retrospective, case-note survey of type 2 diabetes patients prescribed incretin-based therapies in clinical practice. Diabetes Ther 4: 27-40.

33. Nauck M, Frid A, Hermansen K, Thomsen AB, During M, et al. (2013) Longterm efficacy and safety comparison of liraglutide, glimepiride and placebo, all in combination with metformin in type 2 diabetes: 2-year results from the LEAD2 study. Diabetes Obes Metab 15: 204-212.

34. Pratley R, Nauck M, Bailey T, Montanya E, Cuddihy R, et al. (2011) One year of liraglutide treatment offers sustained and more effective glycaemic control and weight reduction compared with sitagliptin, both in combination with metformin, in patients with type 2 diabetes: a randomised, parallel-group, open-label trial. Int. J. Clin. Pract 65: 397-407. 